\title{
Remote monitoring and follow-up of cardiovascular implantable electronic devices in the Netherlands
}

\author{
An expert consensus report of the Netherlands Society of Cardiology
}

\author{
C. C. de Cock • J. Elders • N. M. van Hemel • \\ K. van den Broek • L. van Erven • B. de Mol • \\ J. Talmon • D. A. M. J. Theuns $\cdot$ W. de Voogt
}

Published online: 17 January 2012

(C) The Author(s) 2012. This article is published with open access at Springerlink.com

\begin{abstract}
Remote monitoring of cardiac implanted electronic devices (CIED: pacemaker, cardiac resynchronisation therapy device and implantable cardioverter defibrillator) has been developed for technical control and follow-up using transtelephonic data transmission. In addition, automatic or patienttriggered alerts are sent to the cardiologist or allied professional who can respond if necessary with various interventions. The advantage of remote monitoring appears obvious in impending CIED failures and suspected symptoms but is less likely in routine follow-up of CIED. For this follow-up the indications, quality of care, cost-effectiveneness and patient satisfaction have to be determined before remote CIED monitoring can be applied in daily practice. Nevertheless remote CIED monitoring is expanding rapidly in the Netherlands without
\end{abstract}

C. C. de Cock $(\bowtie)$

Department of Cardiology, VU University Medical Center,

Boelelaan 1117,

1081 HV Amsterdam, the Netherlands

e-mail: cc.dcock@vumc.nl

J. Elders

Department of Cardiology, Canisius Wilhelmina Hospital,

Weg door Jonkerbos 100 ,

6500 GS Nijmegen, the Netherlands

N. M. van Hemel

Heart Lung Center, Utrecht University,

P.O. Box 80125, Utrecht, the Netherlands

K. van den Broek

Department of Medical Psychology,

Tilburg University,

Warandelaan 2,

5037 AB Tilburg, the Netherlands professional agreements about methodology, responsibilities of all the parties involved and that of the device patient, and reimbursement. The purpose of this consensus document on remote CIED monitoring and follow-up is to lay the base for a nationwide, uniform implementation in the Netherlands. This report describes the technical communication, current indications, benefits and limitations of remote CIED monitoring and follow-up, the role of the patient and device manufacturer, and costs and reimbursement. The view of cardiology experts and of other disciplines in conjunction with literature was incorporated in a preliminary series of recommendations. In addition, an overview of the questions related to remote CIED monitoring that need to be answered is given. This consensus document can be used for future guidelines for the Dutch profession.

L. van Erven

Department of Cardiology, Leiden University Medical Center,

Albinusdreef 2,

2333 ZA Leiden, the Netherlands

B. de Mol

Department of Cardio-thoracic Surgery, Academic Medical Center,

Meibergdreef 9,

1105 AZ Amsterdam, the Netherlands

J. Talmon

Department of Medical Informatics, Maastricht University,

P.O. Box 616, 6200 MD Maastricht, the Netherlands

D. A. M. J. Theuns

Department of Electrophysiology, Erasmus Medical Center,

Dr. Molewaterplein 40,

3015 GD Rotterdam, the Netherlands

W. de Voogt

Department of Cardiology, Sint Lucas Andreas Hospital,

Tooropsstraat 164,

1961 AE Amsterdam, the Netherlands 
Keywords Consensus · Remote monitoring · Cardiac device

$\begin{array}{ll}\text { Abbreviations } \\ \text { AF } & \text { Atrial fibrillation } \\ \text { CIED } & \text { Cardiovascular implantable electronic device } \\ \text { CRT } & \text { Cardiac resynchronization therapy } \\ \text { DDDR } & \text { Mode of atrial-ventricular cardiac pacing } \\ \text { DICOM } & \text { Digital imaging and communications in medicine } \\ \text { EPR } & \text { Electronic patient record } \\ \text { EHR } & \text { Electronic health record } \\ \text { ERI } & \text { Elective replacement indicator } \\ \text { GPRS } & \text { General packet radio service } \\ \text { GSM } & \text { Global system for mobile communications } \\ \text { GUI } & \text { Graphical user interface } \\ \text { HL7 } & \text { Health level seven, international healthcare standard } \\ \text { https } & \text { Secure internet connection } \\ \text { ICD } & \text { Implantable cardioverter defibrillator } \\ \text { IDCO } & \text { Implantable device-cardiac-observation } \\ \text { IHE } & \text { Integrating the healthcare enterprise } \\ \text { NEN } & \text { Nederlands normalisatie-instituut } \\ \text { NTA } & \text { Nederlandse Technische Afspraak } \\ \text { PM } & \text { Pacemaker } \\ \text { SMS } & \text { Short message service } \\ \text { VVIR } & \text { Mode of ventricular cardiac pacing } \\ \text { VT } & \text { Ventricular tachycardia }\end{array}$

\section{Chapter 1: introduction}

Cardiovascular implantable electronic devices (CIED) are categorised as implantable pacemakers (PM), implantable cardioverter-defibrillators (ICD), cardiac resynchronisation therapy devices with or without a cardiac defibrillator and cardiac arrhythmia monitor devices. After CIED implantation regular technical checks of the CIEDS are mandatory to examine the performance, safety and remaining longevity of the implanted device. In addition, an in-hospital examination of the device recipient (device patient) is sometimes needed to evaluate his/her physical and mental condition and if required, to adjust the function of the CIED to the changing needs of the device recipient. Guidelines recommend the frequency and intensity of these medical and technical follow-up visits. [1, 2]

Recently remote CIED interrogation from a patient's residence has been introduced as a novel method of care in cardiology [3-18]. With transtelephonic methods, technical information is collected about the attributes, settings and status of CIED and where applicable delivery of electrical therapy by CIED and the resulting outcome. Some physiological parameters of the device patient can also be monitored. This remote interrogation can be used for scheduled and unscheduled technical and patient monitoring and follow-up. This information can result in alerts that may require a response from the cardiologist or allied professional.
How remote monitoring contributes to patient care has been reported in several recent studies. $[9,15,17,18]$ However the indications, clinical relevance, cost effectiveness, management and organisation of remote monitoring are hardly established by prospective randomised trials. [16, 19-21] In addition many organisational and legal aspects as well as reimbursement are still a matter of dispute. Consequently remote monitoring of CIED is now carried out in a very variable and fragmented way in daily practice and lacks a uniform methodology, thus creating uncertainty for all the parties involved. [22]

To overcome this uncertainty it is crucial to make an inventory of all the components of remote monitoring in order to promote a uniform methodology in clinical practice. This consensus report intends to elucidate these components and explores the pitfalls hampering a standard care of device patients in terms of quality, technical and legal issues, safety, costs and reimbursement. In the absence of a high level of evidence (class 1), cardiology experts and those from various disciplines contributed to the advisories and conclusions of this consensus report. These efforts resulted in a preliminary series of recommendations for remote monitoring. This consensus report aims to become the founding base for future guidelines for remote monitoring of CIED to develop in the Netherlands. [23]

Footnote 1. Although the acronym AIMD (Active Implantable Medical Device) is used in the Medical Device Directive 93/42/EEC, in this document the term CIED (Cardiovascular Implantable Electronic Device) is employed, as this term limits the AIMDs to those used only for cardiovascular application.

Footnote 2. Remote CIED monitoring potentially enables reprogramming of operational device algorithms, e.g. to adapt pacing features. Although this approach reflects true 'remote cardiac rhythm management' its application does not appear advisable at this moment due to unknown safety issues and legal uncertainties and needs to be investigated.

\section{Chapter 2: technical description of remote monitoring}

The communication chain

Remote monitoring is capable of sending information from a CIED about programmable features, information on device function, arrhythmias, cardiovascular haemodynamic information, patient clinical status and registrations of stored electrograms to an external device, the transmitter. This transmitter has to be in the proximity of the patient to be able to communicate with the CIED. When the transmitter has received the data stream from the CIED, it sends these data encrypted to a central server of the manufacturer of the CIED.

The manufacturer's device provides these data in a protected environment for the cardiologist or allied professional; 
these data are suitable for incorporation in the hospital information system (HIS). In contrast to in-hospital continuous cardiac rhythm monitoring, in remote monitoring the CIED only communicates with the transmitter at scheduled times, e.g. once a day.

In case of special events detected by the CIED or triggered by the patient, the transmitter will send a nonscheduled transmission to the server, which will be delivered to the cardiologist or allied professional by e-mail and/ or short message service (SMS). When no abnormalities are detected, a transmission occurs as a scheduled agenda-based virtual follow-up. Although the CIED continuously collects data, the transmitter will only send the data according to scheduled time intervals, e.g. once every day. Thus in a technical sense, no continuous 24 h/day, 7 days/week monitoring of the device and/or of the CIED patient has been possible until now. Device companies cannot be held responsible for delays or lack of alerts and follow-up due to a failing landline or global system for mobile communications (GSM) technology.

Communication between CIED and transmitter (Fig. 1)

To remotely monitor a patient, the data from the CIED are transferred to the hardware installed at the patient's home. The initial method to communicate with the CIED is the classic induction coil, known as the programming wand. The coil has to be placed within centimetres over the implanted device. This communication depends on patient compliance. Today's CIEDs are equipped with bidirectional radio-frequent (RF)

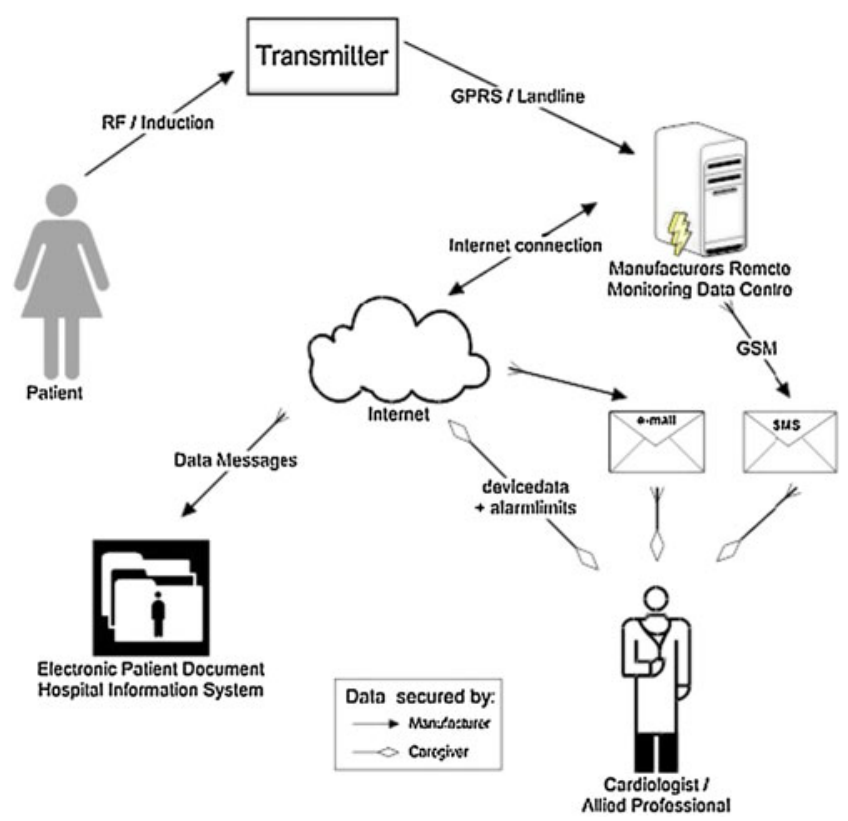

Fig. 1 Global overview of remote monitoring and remote follow-up data flow communication, operating in the frequency range between $403 \mathrm{MHz}$ and $406 \mathrm{MHz}$. With the use of this radio technique, data are automatically sent from the CIED to a local transmitter in the direct proximity of the patient.

Communication between the transmitter and the manufacturer's remote monitoring data centre

The data are forwarded to the manufacturer's remote monitoring data centre. A transmitter is used to receive, amplify and transmit the signals. The data can be sent to the manufacturer's remote monitoring data centre by the conventional telephone cable network often called a 'landline' or by a digital cellular phone technology such as general packet radio service (GPRS) or satellite. All communication between the transmitter and the manufacturer's data centre should be encrypted to guarantee patient privacy and safety [24].

Communication between the manufacturer's data centre and the cardiologist

The raw data are received in the manufacturer's remote monitoring data centre where the data are automatically transformed to a format that is readable for the cardiologist and allied professional using the internet.

Footnote 3: In this report this device is designated as a transmitter rather than the more appropriate but unfamiliar, generic term transponder

The manufacturer's graphical user interface (GUI) permits scrolling through the stored data to allow analysis by the cardiologist or allied professional. The presented data have an identical format to the programmer-based interrogation during an in-hospital visit. In addition the manufacturer's system analyses and compares the data with programmed values to produce alerts. Alarm messages are sent by e-mail or by digital cellular phone short message service (SMS).

Interoperability

To promote an integral follow-up of the device patient, all information including remote monitoring data should be instantaneously and centrally available to the cardiologist or allied professional. Interoperability in remote CIED monitoring is therefore imperative. To ensure this approach, the technology and contents of the information must be exchangeable by applying standards on various levels of processing, communication and semantics.

The ultimate target is the avoidance of any handwritten file but direct automated reading and filling out of data from the patient at home to the electronic patient document (EPD) of HIS saving time and preventing flaws. 
Communication between the manufacturer's data centre and the hospital information systems

Patient data should be regarded as part of the patient file and should be stored in the HIS. Nowadays differences exist between the format and screen displays of the device manufacturers and the local HIS. This difference inhibits a uniform approach to storing and displaying remote monitoring data. Efforts should be made to implement, on a nationwide scale, the Integrating the Healthcare Enterprise (IHE) specification of Implantable Device-Cardiac-Observation (IDCO) for data from the CIED. This specification together with the IEEE 11073-10103-MDC-IDC Nomenclature constitutes the foundation for exchange.

\section{Communication between the manufacturer's data centre and the national health information technology infrastructure}

The concept of the national health IT infrastructure aims to connect all well-maintained health information systems to this infrastructure. The main component is the switch point where an index of the location of record fragments of the patient is maintained. Any accredited health care professional is able to query the switch point for certain types of information. The system will retrieve the requested information from the sources that are linked to the switch board. This infrastructure could potentially also be used to make CIED data available for authorised cardiologists and allied professionals and hospitals. The National Cardiac Devices Registry (NCDR), founded by the Netherlands Society of Cardiology, can contribute to this objective.

\section{Chapter 3: indications, benefits and limitations of remote CIED monitoring}

Types of remote monitoring

Scheduled transmission of programmed alerts of either device-related (e.g. abnormal lead resistance) or patientrelated (e.g. onset of atrial fibrillation) events from the manufacturer's remote monitoring system to the cardiologist is most frequently applied at the moment. This type of remote monitoring includes detection and mailing of events that had initially been programmed by the cardiologist or allied professional. The second type is transmission of a patient-activated alert that indicates the occurrence of specific symptoms. The third type is "remote follow-up" defined as remote device interrogation at scheduled intervals. This method can partly replace the in-hospital technical CIED control.
Indications for remote monitoring of implanted devices

Literature shows a wide spectrum of reasons to perform remote CIED monitoring. [7, 8, 10-15, 17, 18, 25, 26] The reason for CIED implantation, the severity of illness of the device patient, and the opportunity to follow high-risk CIED or field actions/safety alerts of devices appear beneficial indications. [27] For devices and/or leads with a high risk of technical malfunction or great technical complexity (e.g. ICD) remote monitoring appears favourable. [28] Remote monitoring can also be applied to document the underlying cause of symptoms of the device patient comparable with Holter monitoring. Because of the lack of evidence on efficiency or cost-effectiveness, the indications for remote CIED monitoring are currently hardly established and therefore routine remote monitoring of patients with PM, CRT or ICD should be evaluated in further studies. $[2,4,6,16]$ If remote monitoring is applied, the cardiologist's decision is based on assumed better patient care and/or quality of life and tailored to the individual patient.

Timing of response to alerts

As stated in the technical description (see chapter 2) routine remote CIED monitoring cannot offer a continuous surveillance of the device patient. Consequently a 24 h/day, 7 days/ week alert response from the cardiologists to the device patient is not technically feasible. Furthermore evidence of cost-effectiveness of continuous remote monitoring in terms of an immediate response of the cardiologist or allied professional to the alerts is hardly documented [3, 6, 16, 21].

In-hospital visits additional to remote CIED monitoring

Current guidelines for CIED follow-up advise on methods and frequency of technical controls. For conventional follow-up at least one visit per year for the simplest singlechamber PM and 6 monthly follow-up for the more complex dual-chamber PM is advised whereas for the ICD patient after the 1st implantation or replacement a more frequent follow-up is recommended. [1] In congestive heart failure and CRT the course of the failing heart or incidence of arrhythmia determine the intervals of in-hospital visits [1]. Regarding remote CIED monitoring it is recommended that any patient with a CIED is assessed in person at least once a year. [2] However the frequency of the in-hospital visits has to be left to the cardiologist's discretion.

\section{Benefits of remote CIED monitoring}

The major advantage of remote CIED monitoring is the timely detection of CIED failures or deviations of the programmed device values. A fast response to mailed alerts 
by the cardiologist or allied professional can prevent potential harm to the device patient. [8, 10, 12, 13, 15, 17, 18, 25] Secondly, scheduled remote CIED follow-up can save the time and efforts of the cardiologist and allied professional and of the device patient and the accompanying family by avoiding in-hospital visits for technical CIED follow-up. Thirdly, remote CIED monitoring can be used for temporary purposes such as rapid detection of technical failures related to field action/safety alert or avoidance of frequent controls at the time of anticipated battery depletion of the implanted device. Fourthly, it is expected that remote monitoring can counteract the pending imbalance between the annually increasing load of care in more, older and more complex CIED patients [29-31].

\section{Limitations of remote monitoring}

Remote CIED monitoring delivers a substantial amount of data to be handled by the cardiologist and allied professional. This overflow is intrinsically related to continuous monitoring and storage of CIED measurements as well as to stored cardiac and biological signals of the device patient that are transmitted to the manufacturer's remote monitoring centre at scheduled intervals. Their clinical relevance is often unknown in terms of risk and prognosis. This information may falsely initiate the prescription of drugs or force unnecessary CIED reprogramming and hospital referral leading to over-treatment.

Apart from the clinical consequences, the overflow of data generated by remote monitoring may increase the daily workload of the cardiology staff thus requiring more personnel and budget. Remote monitoring might unfavourably affect the attitude of the device patient because this approach can be falsely appreciated as an alternative route to unrestricted and immediate access to care. Furthermore, alerts and other information resulting from remote monitoring may disturb the mental condition and health perception of the device recipient. [32-34] These potential disadvantages require further specific studies. Anticipating the results of these studies, one should accept that alerts and responses to alerts are reserved for specific conditions such as technical failures or abnormalities of CIED function specified in the monitoring protocol of the individual device recipient

Measurements of quality of care of remote monitoring

Because of the novelty of this type of patient care, which elicits uncertainty about benefit, adverse effects and costs, measures to determine the quality of care are urgently needed. Clinical and technical characteristics that predict potential benefit of remote monitoring have to be developed. The set of recommendations delivered in this document by experts can serve as a standard (see chapter 7). Current technology of transmission, storage, alerts and communication between the manufacturer's remote monitoring centre, cardiologist and allied professional and HIS will further develop and these products should also be incorporated in the predictors of benefit.

\section{Conclusions}

1. Remote CIED monitoring can be applied but its indications are hardly scientifically established. Therefore remote monitoring cannot be considered obligatory patient care and is left at the cardiologist's discretion in daily practice.

2. From the technical point of view remote CIED monitoring does not imply continuous monitoring but scheduled or unscheduled information to the cardiologist or allied professional. Until now evidence is lacking to justify 24 h/day, $/ 7$ days/week based remote monitoring. Waiting for clear evidence, handling of remote monitoring should be restricted to hospital office hours.

3. Considering the benefits and limitations of remote monitoring, prospective comparative studies are needed to assess the contribution of remote CIED monitoring to the care of the device patient. The cost-effectiveness of the indications for various CIED categories and predictors of benefit should be assessed.

4. Technical developments of remote monitoring require interaction with manufacturers to facilitate uniform methods of care and guarantee clinical relevance of remote monitoring.

\section{Chapter 4: the role of the patient in remote monitoring}

\section{Patient participation}

Remote CIED monitoring relies on regular contact between the device recipient and transmitter at scheduled intervals (e.g. at home during the night). Therefore the device recipient must be in the proximity of the transmitter. Patient compliance includes matching of mental functioning as well as the visual and hearing capacities of the device patient to proper handling of the communication system. Remote monitoring also relies on patient accessibility for alerts from the hospital and counselling by the cardiologist and allied professional.

\section{Continuous surveillance}

The device patient might perceive that remote CIED monitoring brings out a continuous surveillance of the implanted device and cardiac rhythm. However, as stated in chapter 2, transmitting of surveillance data occurs at regular intervals and not continuously. The same holds for delivery of alerts 
to the hospital. A delay in receiving a transmission can occur which rules out continuous monitoring.

The internet communication can be falsely perceived as an alternative route to immediate or easy access to patient care in the hospital or emergency department. However, alerts and response to alerts have to be reserved for specific conditions, technical failures and particular appointments with the patient. A commitment of the patient to the appropriate use of remote CIED monitoring is therefore indispensable (see $7 \mathrm{~b}$ Patient management).

\section{Responsibilities}

Patients receiving remote CIED monitoring should accept responsibilities for proper handling and care of the transmitter, prevention of damage and measures for proper communication with the device centre. These aspects are already formalised by current legal rules of ownership, in terms of "good clinical practice". [35] Information to the device patient should point to these responsibilities regarding all parts of remote monitoring and follow-up. In case of serious acute events the patient should ask for care in the conventional way.

\section{Ownership of the transmission system}

Until now, the payment for the transmitter and additional tools has varied widely and markedly. The total cost has until now been compensated by the health insurance company. Hospital variations occur in the payment of transmitter hardware. Sometimes the cost of the transmitter is included in the device costs but in other circumstances the transmitter is charged separately. Therefore a uniform format for reimbursement of all facilities for remote monitoring, namely incorporation in the reimbursement structure, is strongly advocated.

Transmitters are paired to a unique CIED and therefore they are not exchangeable between patients. This inhibits the transfer or loaning of the transmitter to other device patients, e.g. after death. Technical facilities for exchange of the transmission systems can promote flexibility of remote monitoring. If patient ownership were to be abandoned, the hospital could become the owner of the transmission systems. It is, however, unclear whether this transition would reduce investments and maintenance time.

\section{Conclusions}

1. The implementation of remote CIED monitoring in hospitals also involves patient information about the expectations and restrictions of this method, and the contribution and responsibilities of the device patient.
2. The ownership of the transmitting systems by the hospital can promote the flexibility of remote monitoring for reuse but it is unclear whether this approach will reduce the overall costs.

3. Organisations of CIED patients should be asked to participate in the provision of patient information about these subjects in order to facilitate a good perception of the advantages and disadvantages of remote CIED monitoring.

4. It is advised to compose a written consent between the hospital or cardiologist and the patient regarding the respective responsibilities, duties and limits of remote CIED monitoring. Several subjects should be addressed such as the responsibility for the analysis of the transmitted data, timing of analysis of transmitted data and feedback to the patient, and regulations about unscheduled transmissions.

\section{Chapter 5: the contribution of the device manufacturer to remote monitoring}

Introduction

The development of remote CIED monitoring was started many years ago initially to employ technical follow-up by telephone. $[25,26,36,37]$ For the device manufacturer remote monitoring also constituted a tool to follow devices that were assigned as field action/safety alert or high risk for sudden failure. Furthermore data of remote monitoring can be used after informed patient consent for assessment of long-term CIED performance contributing to improved post-market CIED surveillance.

The market competition of the device branch has resulted in a large variety of tools due to differences in design, presentation of collected data, programming facilities and communication features. Such a variety may have a negative impact on patient safety as clinicians may get confused when they deal with different manufacturers that handle critical issues in different ways. In the past the responsibilities of the CIED manufacturers with regard to security and confidentiality resulted in a series of USA and European regulations. However, implementation of remote CIED monitoring clearly enlarges the manufacturer's responsibilities and these should be clarified. These aspects and the manufacturer's interaction with the cardiologist, allied professional and hospital also need to be formalised.

\section{Privacy}

Transmitted data and alerts may be communicated by the device provider through e-mail or by telephone or SMS and received in the hospital. These messages must be encrypted to guarantee patient privacy. Access to manufacturers' 
websites to assess CIED data of specific patients should also take place in a secure way (https).

\section{Safety}

The accuracy of data receipt in the manufacturer's remote monitoring centre, data transmission to the hospital and priorities with respect to alerts are the manufacturer's responsibility. The manufacturer is responsible for the $24 \mathrm{~h} /$ day, 7 days/week availability of service but can make agreements about a temporary gap in monitoring for specific reasons.

However the manufacturer cannot be held responsible for failures due to insufficient patient instruction delivered by the cardiologist or allied professional or the misinterpretation of those instructions by the patient. This also applies for failed transmission, temporary delay or missing alerts due to disorders of landline or GSM technology; in principle telecom service providers are responsible for this part of the communication link, though the manufacturer has the responsibility to select a reliable provider.

Because remote monitoring is susceptible to computer security breaches of various origins, performance, safety and privacy are at stake, requiring specific security controls. A minimum format for secure data transport is mandatory for both manufacturer and hospital environment. [25]

Storage of technical and patient data

The manufacturer is not the owner of the patient data. Patient data should be regarded as part of the patient file and therefore should be stored for a limited time in the manufacturer's remote monitoring system; appropriate back-up of all data is needed. Patient data should be treated according standard rules (NEN 7510).

Storage of patient data ends after final transmission of data to the hospital where storage is obliged over a 15 -year interval. [38] If the manufacturer wants to analyse or store patient data longer, non-reversible transformation into an unidentifiable format is necessary.

\section{Technical developments}

The current technology of remote monitoring will further develop by data reduction, communication with electronic patient records and personal health records and by adding more features. These developments require interaction between CIED manufacturers and health care providers to promote uniform methodology and to evaluate the clinical relevance of technical progress. To avoid clinically irrelevant applications of remote CIED monitoring, interaction between cardiology professionals and CIED manufacturers should be intensified.

\section{Conclusions}

1. Regarding CIED remote monitoring, the responsibilities of the device manufacturer and the local cardiology professionals and hospital, respectively, should be formalised in a written agreement. This document covers the technical, safety and privacy matters addressed in this chapter as well as agreements about data storage and mutual rights and agreements.

2. Uniformity in the methods of remote CIED monitoring as well as standardised presentation of data ranks very highly in the regulations to be developed between device manufacturers and cardiology professionals.

3. Today's regulations for safety and privacy of CIED applicable to CIED manufactures have to be revisited with the employment of remote CIED monitoring, particularly to prevent computer breaches of the device.

\section{Chapter 6: costs and reimbursement of remote monitoring}

Cost components for the hospital

As shown in Fig. 1 (see chapter 2), the transmitter at the patient's residency, the manufacturer's information technology, terminals in the hospital and the HIS constitute linking parts. The total cost of setting up the system, providing care and maintaining the system can be divided into different aspects:

Initial investments:

- Time and budget to connect the incoming data and alerts to the HIS and to maintain these connections and the database,

- The hospital and its cardiology department have to reserve technical and human facilities for remote monitoring and storage of the data,

- Organisational efforts,

- During the initial starting phase, the care providers have to familiarise themselves with the system and need to support the conventional follow-up.

Resource use:

- Reading and interpretation of the alerts or scheduled remote follow-up,

- Patient instruction, telephonic and local checks of the chain of transmission often at the patient's residency,

- Response to and reassurance and counselling of the patient at his residency by information about the results,

- Administrative assistance for patient filing and storage of data. 
Capital equipment:

- The transmitter at the patient's residence.

Maintenance:

- Maintenance of the web-application/provider,

- Maintenance of the database,

- Maintenance of the physical system at the patient's residence.

To date the purchase of the transmitter and associated technical facilities as well as transmission of data and alerts are usually free of charge for the patient.

\section{Reimbursement of conventional CIED follow-up}

Follow-up after CIED implantation and replacement carried out according to current guidelines [1,2] reimbursed by insurance companies applying standard reimbursement rules for this care. The follow-up includes at least one in-hospital medical consultation and a technical check of CIED of which the details are published at the website of $D B C$ Onderhoud (www.dbconderhoud.nl).

\section{Reimbursement of remote CIED monitoring}

Until now remote CIED monitoring has not been reimbursed by health insurance companies. [9, 29, 30] In the absence of Dutch advisories or guidelines for remote CIED monitoring, health insurance companies can refuse reimbursement to the cardiologist, hospital and manufacturer and can ask for supplementary payment by the device patient. On the other hand with the purpose to improve quality of care, insurance companies and health authorities can propose the application of remote monitoring as the standard method. From the patient perspective, remote monitoring appears very attractive. The initial reports point to a significantly better health perception of the device patient with remote monitoring because of the perceived "short distance care" and easy access to the hospital. [11, 13, 16, 30] This additional gain of health perception therefore justifies additional costs. Furthermore, if remote monitoring becomes an evidence-based type of care for the CIED patient, payment by the device patient for remote monitoring is inappropriate and should be covered by the health insurance company.

The costs for the cardiologists and hospital are completely unknown at the moment because implementation of remote CIED monitoring runs parallel with standard outpatient follow-up and requires extra investments in information technology, more highly qualified personnel and other measures in the initial years. In view of all these matters it is unclear whether the financial balance will end positively; this lack of knowledge requires prospective costeffectiveness studies in the Dutch healthcare setting [19]

It appears logical to incorporate remote CIED monitoring in the existing generic DBC structure. Pilot studies can already be started to examine the impact on time and costs of various technical and human components of remote CIED monitoring as well as the reasons for and frequency of remote monitoring.

Conclusions

1. Remote CIED monitoring is technically feasible and may offer beneficial care in specific indications. However, it has a major impact on time, technical facilities and personnel, and therefore appropriate reimbursement is needed.

2. Reimbursement is preferable through the existing generic reimbursement structure.

3. Short-term pilot studies are requested to evaluate the frequency of and reasons for remote CIED monitoring currently done in the Netherlands to define the degree and level of reimbursement without delay.

4. Cost-effectiveness studies of remote CIED monitoring should be initiated to evaluate the efficiency of various indications for remote monitoring in the Dutch health setting. The results should be used for fine-tuning of the current reimbursement structure.

5. Remote CIED monitoring can become a standard component of CIED patient care with indications based on evidence and guidelines.

\section{Chapter 7: preliminary recommendations for remote CIED monitoring}

a. Technical features

1. Contents of alerts

Initial experience with remote CIED monitoring delivered a series of preliminary recommendations for the content of default or optional programmed alerts. Table 1 recommended minimal device settings in remote monitoring.

2. Application of scheduled remote CIED monitoring

For all CIEDS default and optional alert features can be installed.

PM: Optional alert features include the detection of atrial and ventricular arrhythmia events, percentages of atrial and/or ventricular pacing, rate histograms and sensor driven pacing rhythms; this information can contribute to optimal individualised pacing.

ICD: The detection of VF, VT and all VT types resulting in ICD discharge, aborted shocks and the 
Table 1 Recommended minimal device settings in remote monitoring

\begin{tabular}{|c|c|c|c|c|c|}
\hline Condition & Device & Default & Optional & Scheduled & Unscheduled \\
\hline \multirow[t]{4}{*}{ Battery } & $\mathrm{PM} / \mathrm{ICD} / \mathrm{CRT}$ & Battery Impedance & & $\mathrm{X}$ & \\
\hline & $\mathrm{PM} / \mathrm{ICD} / \mathrm{CRT}$ & Estimated Replacement time (ERT) & & $\mathrm{X}$ & $\mathrm{X}$ \\
\hline & ICD & Capacitor charge time & & $\mathrm{X}$ & \\
\hline & ICD & Capacitor charge time $>18$ seconds & & $\mathrm{X}$ & $\mathrm{X}$ \\
\hline \multirow[t]{4}{*}{ Leads } & & Lead Impedance & & $\mathrm{X}$ & \\
\hline & $\mathrm{PM} / \mathrm{CRT} / \mathrm{ICD}$ & $20 \%$ Deviation of normal values & & $\mathrm{X}$ & $\mathrm{X}$ \\
\hline & ICD & Shock impedance & & $\mathrm{X}$ & \\
\hline & ICD & Shock impedance out of range & & $\mathrm{X}$ & $\mathrm{X}$ \\
\hline \multirow[t]{3}{*}{ Sensing } & $\mathrm{PM} / \mathrm{ICD} / \mathrm{CRT}$ & Intrinsic atrial signals & & $\mathrm{X}$ & \\
\hline & $\mathrm{PM} / \mathrm{ICD} / \mathrm{CRT}$ & Intrinsic ventricular signals & & $\mathrm{X}$ & \\
\hline & $\mathrm{PM} / \mathrm{ICD} / \mathrm{CRT}$ & $20 \%$ Deviation of normal values & Detection of interference & $\mathrm{X}$ & $\mathrm{X}$ \\
\hline \multirow[t]{3}{*}{ Threshold } & $\mathrm{PM} / \mathrm{ICD} / \mathrm{CRT}$ & Automatic atrial capture management & & $\mathrm{X}$ & \\
\hline & $\mathrm{PM} / \mathrm{ICD} / \mathrm{CRT}$ & Automatic ventricular capture management & & $\mathrm{X}$ & \\
\hline & $\mathrm{PM} / \mathrm{ICD} / \mathrm{CRT}$ & $20 \%$ Deviation of normal values & & $\mathrm{X}$ & $\mathrm{X}$ \\
\hline \multirow[t]{2}{*}{ Programming } & ICD & VT/VF therapy disabled & & $\mathrm{X}$ & \\
\hline & $\mathrm{PM} / \mathrm{ICD} / \mathrm{CRT}$ & Device reset & & $\mathrm{X}$ & $\mathrm{X}$ \\
\hline \multirow[t]{9}{*}{ Arrhythmias } & $\mathrm{PM} / \mathrm{ICD} / \mathrm{CRT}$ & & Incidence & $\mathrm{X}$ & \\
\hline & $\mathrm{PM} / \mathrm{ICD} / \mathrm{CRT}$ & & Duration & $\mathrm{X}$ & \\
\hline & $\mathrm{PM} / \mathrm{ICD} / \mathrm{CRT}$ & & Supraventricular events & $\mathrm{X}$ & \\
\hline & $\mathrm{PM} / \mathrm{ICD} / \mathrm{CRT}$ & Duration of AF exceeds consented time & & $\mathrm{X}$ & $\mathrm{X}$ \\
\hline & $\mathrm{PM} / \mathrm{ICD} / \mathrm{CRT}$ & & Ventricular events & $\mathrm{X}$ & $\mathrm{X}$ \\
\hline & ICD & VT Therapy & & $\mathrm{X}$ & $\mathrm{X}$ \\
\hline & ICD & VF therapy & & $\mathrm{X}$ & $\mathrm{X}$ \\
\hline & ICD & More than 3 therapies needed for termination & & $\mathrm{X}$ & $\mathrm{X}$ \\
\hline & ICD & Therapy not successful & & $\mathrm{X}$ & $\mathrm{X}$ \\
\hline \multirow[t]{4}{*}{ Heart rate } & $\mathrm{PM} / \mathrm{ICD} / \mathrm{CRT}$ & & $\%$ Atrial and ventricular pacing & $\mathrm{X}$ & \\
\hline & & & $\%$ Atrial and ventricular sensing & $\mathrm{X}$ & \\
\hline & & & Rate histograms (intrinsic) & $\mathrm{X}$ & \\
\hline & & & Sensor driven rate histograms & $\mathrm{X}$ & \\
\hline \multirow[t]{2}{*}{ Heart failure } & $\mathrm{PM} / \mathrm{CRT} / \mathrm{ICD}$ & & Heart failure monitoring parameters & $\mathrm{X}$ & $\mathrm{X}$ \\
\hline & CRT & $\%$ biventricular pacing & & $\mathrm{X}$ & $\mathrm{X}$ \\
\hline
\end{tabular}

number of appropriate and inappropriate shocks is indispensable to get insight into ICD functioning. Monitoring of the intrinsic ventricular signal for sensing has a high priority.

CRT: The percentage of biventricular ventricular pacing is a paramount feature of CRT functioning.

3. Triggered event monitoring: For all CIEDs specific default and optional alert features can be installed as advised for scheduled monitoring.

4. Comments on contents of remote monitoring:

4a. Programming of the alert feature for the event VT requires a definition of this arrhythmia. According to the expert view VT is defined as a rate $>180 / \mathrm{m}$ for $30 \mathrm{~s}$ because this pattern is associated with symptoms and risk. 4b. Programming of the alert feature for the impact of $\mathrm{AF}$ requires a definition of this arrhythmia. According to expert view, mode switch of the pacing device after AF detection to VDIR or DDIR $>10 \%$ of day time and $>2$ days monthly is classified as a serious AF incidence. Since inappropriate mode switch has been reported additional conformation is required. [39]

4c. It is advocated not only to rely on actual data of the alarm but also to get insight into trends of the concerning alarms, e.g. course of lead resistance.

4d. Not only the actual value of a measurement, e.g. abnormal battery impedance, but also facilities for comparing previous data permitting a trend evaluation are strongly recommended. 
7b. Patient management

Because written information is a crucial factor for a safe and accurate remote CIED monitoring and followup, it is advised to formulate an outline with minimal requirements of information for nation-wide use. The following elements should be addressed:

1. The device patient should be informed in such a way that he/she is aware that regular follow-up by remote monitoring is a safe and convenient way to replace some in-hospital visits. Remote CIED monitoring is not a continuous process but has to be limited to the "office-hours" of the hospital. The information should include that alerts will be responded to within a reasonable timeframe (e.g. in the day of receipt). The patient and provider should be familiar with this timing to avoid any misunderstanding.

2. The timing of response to an alert (patient and physician response time respectively) should be agreed between the patient and the cardiologist and allied professional. Regulations should be agreed on how to reach the patient and the timing necessary to warn the device patient after a received alert. The impact of alerts resulting in any action (sometimes hospital referral) should be addressed. Since the stored data are part of the medical file, the patient should be permitted to inspect his/her own data.

3. Patients with remote CIED monitoring need to be informed about the results of the technical followup of the device and/or consequences of alerts. The frequency, intensity and timing of this information should be agreed between the cardiologist and allied professional and the device patient.

4. Consequences of the ownership of the equipment for remote CIED monitoring need to be clarified. The proper handling, consequences of damage and misuse of the transmission equipment need to be addressed.

5. The patient should be informed about the outcome of remote monitoring of his/her device. Furthermore, measures to prevent abuse of remote monitoring or unnecessary referral to the hospital can be given.

6. The patient should inform the cardiologist or allied professional in case of an absence of more than 20 days or any differently agreed interval to maintain the communication between provider and cardiologist and allied professional.

7. Electrical discharges, whether appropriate or not, will trigger alerts or evoke patient activated alerts. The ICD patient should be aware that these alerts can have major consequences, e.g. the temporary withdrawal of the driving license.

8. The responsibility and contribution of the device manufacturer and internet provider for the execution of remote CIED monitoring should be clarified.

9. The information about remote monitoring of implanted devices is to be presented in easily understandable text and the cardiologist or allied professional and device patient have to sign that the information has been given orally and that written information has been received. The oral information can be given per patient or in groups of device patients.

7c. The in-hospital protocol of remote CIED monitoring:

It is advised to compose an in-hospital protocol for remote CIED monitoring to describe the tasks and responsibilities of the cardiologist, allied professional and hospital management. [40] This protocol contains the indications for remote monitoring, programming of alerts and technical, organisational and administrative components of remote monitoring. It is advised to formulate an outline with minimal requirements for nationwide use, containing the following elements:

1. To perform remote monitoring specific cardiac knowledge, skills and experience of cardiac arrhythmias and CIEDs are necessary for the cardiologist and allied professional. Hospitals with a licence to perform ICD implantations and hospitals without this licence but with an agreement with a licensed hospital can perform remote ICD monitoring and follow-up, provided clear written rules on responsibilities are specified. Hospitals without a licence to perform ICD implantations have to meet the same rules and regulations concerning ICD follow-up according to the Dutch Guidelines for ICD implanting centres. [41] All hospitals are free to execute remote monitoring of PM and CRT.

2. The cardiologist decides on the indication, the programming of alerts, frequency and duration of remote CIED monitoring. The cardiologist can transfer these activities to allied professionals according to the standing in-hospital protocol. $\mathrm{Mu}-$ tual interaction between cardiologist and allied professional is necessary to promote safety and relevance of programming and of responses to alerts.

3. The protocol also addresses the written information to the patient, and the priorities and logistic routes to cope with alerts and responses to alerts. The protocol should also underscore that alerts and other remote monitoring data will be responded to within a reasonable time. 
4. To avoid loss of patient information the communication between the remote monitoring chain and the hospital EPD should be encouraged. The cardiology department and the board of the hospital should supervise the contents, quality and implementation of this in-hospital protocol and the written information to the patient.

5. The protocol requests regular upgrading and can serve as a measure to assess the quality of care of device patients in the hospital.

\section{Addendum}

Technical standards for remote CIED monitoring

\section{Technical standards}

The Netherlands Standardization Institute (NEN) has drafted a Nederlandse Technische Afspraak (NTA) which, besides defining Telemedicine, covers the quality aspects of Telemedicine. This NTA is now being revised and will become a Dutch national standard. It will contain requirements for telemedicine providers to develop a quality system that will monitor the risks associated with the service. As soon as this standard becomes available it is advisable to apply this standard for the remote monitoring and remote follow-up of CIEDs.

\section{Quality of the data communication}

Transmitted data are labelled to the unique serial number of the implanted device to prevent patient data being mixed between patients. Data integrity and communication between device, transmitter and the manufacturer's remote monitoring data centre, are the responsibility of the manufacturer. Data encryption is thought to be energy consuming. Encryption is done by the transmitter. The frequent interrogation of the device is battery consuming (estimated 3 months of a lifetime).

Regarding the access of data stored at the manufacturer's remote monitoring data centre, mails and SMS messages should all comply with the Code of Practice for Information Security Management.

3. Currents standards for connectivity can be assigned in three levels

The functional specifications of interconnectivity can be found in documents published by Integrating the Healthcare Enterprise (IHE). IHE is an initiative by healthcare professionals and industry to improve the way computer systems in healthcare share information. IHE promotes the coordinated use of established standards such as DICOM and HL7 to address specific clinical needs in support of optimal patient care. Furthermore IHE defines and publishes technical frameworks for developers and users.

By defining the syntax of message formats and communication rules DICOM and HL7 defines technical specifications. HL7, which is an abbreviation of Health Level Seven, is a standard for exchanging information between medical applications. This standard defines a format for the transmission of health-related information. "Level Seven" refers to the seventh level of the International Organization for Standardization (ISO) seven-layer communications model for Open Systems Interconnection (OSI)-the application level. Digital Imaging and Communications in Medicine (DICOM) is a standard for handling, storing, printing, and transmitting information in medical imaging.

For interoperability it is important that, after sending an observation taken by the implanted device, the semantics of the observation are clearly defined. IEEE 11073-10103 characterises clinical terms by defining correct descriptions and definitions. It provides the nomenclature for the clinical observations taken by CIEDs.

Technical References

1.NEN 7504:2004 Medische Met vriendelijke groet, informatica-Berichtenverkeer-Zorginformatiesystemen-Toepassing van de HL7-berichtenstructuur in zorginstellingen in Nederland-Eisen en toelichting van HL7-versie 2.4; 2. Nen 7510, 2004. Informatiebeveiliging in de zorg. May 2005 at http://www.nen7510.org 3. ISO/HL7 27931:2009. DataExchange Standards-Health Level Seven Version 2.5 -An application protocol for electronic data exchange in healthcare environments 4.. ISO/TS 13606:2009. Electronic health record communication

Open Access This article is distributed under the terms of the Creative Commons Attribution Noncommercial License which permits any noncommercial use, distribution, and reproduction in any medium, provided the original author(s) and source are credited.

\section{References}

1. Vardas PE, Auricchio A, Blanc JJ, et al. Guidelines for cardiac pacing and cardiac resynchronization therapy: the Task Force for Cardiac Pacing and Cardiac Resynchronization Therapy of the European Society of Cardiology. Developed in collaboration with the European Heart Rhythm Association. Eur Heart J. 2007;28 (18):2256-95.

2. Wilkoff BL, Auricchio A, Brugada J, et al. HRS/EHRA expert consensus on the monitoring of cardiovascular implantable electronic devices (CIEDs): description of techniques, indications, personnel, frequency and ethical considerations. Hear Rhythm. 2008;5(6):907-25.

3. Al Khatib SM, Piccini JP, Knight D, et al. Remote monitoring of implantable cardioverter defibrillators versus quarterly device interrogations in clinic: results from a randomized pilot clinical trial. J Cardiovasc Electrophysiol. 2010;21(5):545-50. 
4. Fauchier L, Sadoul N, Kouakam C, et al. Potential cost savings by telemedicine-assisted long-term care of implantable cardioverter defibrillator recipients. Pacing Clin Electrophysiol. 2005;28 Suppl 1:S255-9.

5. Halimi F, Clementy J, Attuel P, et al. Optimized post-operative surveillance of permanent pacemakers by home monitoring: the OEDIPE trial. Europace. 2008;10(12):1392-9.

6. Heidbuchel H, Lioen P, Foulon S, et al. Potential role of remote monitoring for scheduled and unscheduled evaluations of patients with an implantable defibrillator. Europace. 2008;10(3):351-7.

7. Lazarus A. Remote, wireless, ambulatory monitoring of implantable pacemakers, cardioverter defibrillators, and cardiac resynchronization therapy systems: analysis of a worldwide database. Pacing Clin Electrophysiol. 2007;30 Suppl 1:S2-S12.

8. Lunati M, Gasparini M, Santini M, et al. Follow-up of CRT-ICD: implications for the use of remote follow-up systems. Data from the InSync ICD Italian Registry. Pacing Clin Electrophysiol. 2008;31(1):38-46.

9. Morichelli L, Ricci RP. Remote monitoring of implantable devices: the European experience. Hear Rhythm. 2009;6(7):1077-80.

10. Nielsen JC, Kottkamp H, Zabel M, et al. Automatic home monitoring of implantable cardioverter defibrillators. Europace. 2008;10(6):729-35.

11. Raatikainen MJ, Uusimaa P, van Ginneken MM, et al. Remote monitoring of implantable cardioverter defibrillator patients: a safe, time-saving, and cost-effective means for follow-up. Europace. 2008;10(10):1145-51.

12. Ricci RP, Morichelli L, Santini M. Home monitoring remote control of pacemaker and implantable cardioverter defibrillator patients in clinical practice: impact on medical management and health-care resource utilization. Europace. 2008;10(2):164-70.

13. Ricci RP, Morichelli L, Quarta L, et al. Long-term patient acceptance of and satisfaction with implanted device remote monitoring. Europace. 2010;12(5):674-9.

14. Santini M, Ricci RP, Lunati M, et al. Remote monitoring of patients with biventricular defibrillators through the CareLink system improves clinical management of arrhythmias and heart failure episodes. J Interv Card Electrophysiol. 2009;24(1):53-61.

15. Saxon LA, Hayes DL, Gilliam FR, et al. Long-term outcome after ICD and CRT implantation and influence of remote device followup: the ALTITUDE Survival Study. Circulation. 2010;122 (23):2359-67.

16. Schmidt S, Schuchert A, Krieg T, et al. Home telemonitoring in patients with chronic heart failure: a chance to improve patient care? Dtsch Arztebl Int. 2010;107(8):131-8.

17. Theuns DA, Rivero-Ayerza M, Knops P, et al. Analysis of 57,148 transmissions by remote monitoring of implantable cardioverter defibrillators. Pacing Clin Electrophysiol. 2009;32 Suppl 1:S63-5.

18. Varma N, Michalski J, Epstein AE, et al. Automatic remote monitoring of implantable cardioverter-defibrillator lead and generator performance: the Lumos-T Safely RedUceS RouTine Office Device Follow-Up (TRUST) trial. Circ Arrhythm Electrophysiol. 2010;3(5):428-36.

19. Goff-Pronost M, Sicotte C. The added value of thorough economic evaluation of telemedicine networks. Eur J Health Econ. 2010;11 (1):45-55.

20. Hailey D, Ohinmaa A, Roine R. Study quality and evidence of benefit in recent assessments of telemedicine. J Telemed Telecare. 2004;10(6):318-24.

21. Joglar JA. Remote monitoring of cardiovascular implantable electronic devices: new questions raised. Pacing Clin Electrophysiol. 2009;32(12):1489-91.

22. Halimi F, Cantu F. Remote monitoring for active cardiovascular implantable electronic devices: a European survey. Europace. 2010;12:1778-80.
23. van Hemel NM. Remote monitoring of implanted cardiac devices: a plea for a nationwide exploration. Neth Heart J. 2009;17 (11):434-7.

24. Maisel WH, Kohno T. Improving the security and privacy of implantable medical devices. N Engl J Med. 2010;362(13):11646 .

25. Res JC, Theuns DA, Jordaens L. The role of remote monitoring in the reduction of inappropriate implantable cardioverter defibrillator therapies. Clin Res Cardiol. 2006;95 Suppl 3:III17-21.

26. Schoenfeld MH, Compton SJ, Mead RH, et al. Remote monitoring of implantable cardioverter defibrillators: a prospective analysis. Pacing Clin Electrophysiol. 2004;27(6 Pt 1):757-63.

27. Maisel WH, Moynahan M, Zuckerman BD, et al. Pacemaker and ICD generator malfunctions: analysis of Food and Drug Administration annual reports. JAMA. 2006;295(16):1901-6.

28. Borleffs CJ, van Erven L, van Bommel RJ, et al. Risk of failure of transvenous implantable cardioverter-defibrillator leads. Circ Arrhythm Electrophysiol. 2009;2(4):411-6.

29. Merkely B, Roka A, Kutyifa V, et al. Tracing the European course of cardiac resynchronization therapy from 2006 to 2008. Europace. 2010;12(5):692-701.

30. van Veldhuisen DJ, Maass AH, Priori SG, et al. Implementation of device therapy (cardiac resynchronization therapy and implantable cardioverter defibrillator) for patients with heart failure in Europe: changes from 2004 to 2008. Eur J Heart Fail. 2009;11(12):1143-51.

31. Zayac S, Finch N. Recipients' of implanted cardioverterdefibrillators actual and perceived adaptation: a review of the literature. J Am Acad Nurse Pract. 2009;21(10):549-56.

32. Kamphuis HC, de Leeuw JR, Derksen R, et al. Implantable cardioverter defibrillator recipients: quality of life in recipients with and without ICD shock delivery: a prospective study. Europace. 2003;5(4):381-9.

33. Thomas SA, Friedmann E, Gottlieb SS, et al. Changes in psychosocial distress in outpatients with heart failure with implantable cardioverter defibrillators. Heart Lung. 2009;38(2):109-20.

34. van den Broek KC, Denollet J, Nyklicek I, et al. Psychological reaction to potential malfunctioning of implantable defibrillators. Pacing Clin Electrophysiol. 2006;29(9):953-6.

35. Gevers JK. Evaluation of the Dutch Medical Treatment Act (WGBO). Ned Tijdschr Geneeskd. 2001;145(11):509-12.

36. Dreifus LS, Zinberg A, Hurzeler P, et al. Transtelephonic monitoring of 25,919 implanted pacemakers. Pacing Clin Electrophysiol. 1986;9(3):371-8.

37. Platt S, Furman S, Gross JN, Andrews C, Benedek M. Transtelephone monitoring for pacemaker follow-up 1981-1994. Pacing Clin Electrophysiol. 1996;19(12 Pt 1):2089-98.

38. Richtlijnen inzake het omgaan met medische gegevens vastgesteld door de Algemene Vergadering van de KNMG op 15 december 2009. www.knmg.nl/dossier/medischegegevens 2010.

39. de Voogt WG, van Hemel NM. Clinical application of pacemakers in atrial tachyarrhythmias. Neth Heart J. 208;16(Suppl 1):S20 S24.

40. van Eck JW, van Hemel NM, de Voogt WG, et al. Routine followup after pacemaker implantation: frequency, pacemaker programming and professionals in charge. Europace. 2008;10(7):832-7.

41. NVVC praktijkrichtlijn voor ICD centra; implantaties en controles. www.nvvc.nl. 2009.

\section{List of participants}

Organisational and writing committee: $\mathrm{CC}$ de Cock, $\mathrm{MD} \mathrm{PhD}$, Department of Cardiology, VUmc, Amsterdam; J Elders, RN MA, Department of Cardiology, Canisius Wilhelmina Hospital, Nijmegen; NM van Hemel, MD PhD, Prof Em, Utrecht University. 
Subject: technical aspects: $\mathrm{J}$ Talmon, $\mathrm{PhD}$, Department of Medical Informatics, Maastricht University, chairman; MJ van der Ven, Chairman of Project Team Telemedicine NEN; J Versluis, St Jude Medical, Veenendaal; E.T van der Velde, PhD, Department of Cardiology, UMC Leiden; PFHM van Dessel, MD PhD, Department of Cardiology, AMC Amsterdam; P Boogaard, Cygnea, Mijdrecht.

Subject: contents of telemonitoring: DAMJ Theuns, $\mathrm{PhD}$, Department of Cardiology, Erasmus MC Rotterdam, chairman; PP Delnoy, MD PhD, Department of Cardiology, Isala Klinieken Zwolle; P van der Kemp, Sorin/Ela BV, Amsterdam; A Dijk, MSc, Thorax Center, AMC Groningen; YS Tuininga, MD PhD, Department of Cardiology, Deventer Hospital; JH Ruiter, MD PhD, Department of Cardiology, Medical Center Alkmaar; ML Hendriks, MA, Department of Cardiology, VUmc Amsterdam; R Jansen, Department of Cardiology, Medical Center Alkmaar.

Subject: quality of care: $\mathrm{L}$ van Erven, MD PhD, Department of Cardiology, LUMC, Leiden, chairman; W van den Broek, Medtronic Trading NL, Heerlen; B Springorum, Department of Cardiology, Onze Lieve Vrouwe Gasthuis, Amsterdam; A van Kooten, Department of Cardiology, Onze Lieve Vrouwe Gasthuis, Amsterdam; ML Hendriks, MA, Department of Cardiology, VUmc Amsterdam; G Paulussen, MD, Department of Cardiology, Atrium Medical Center, Heerlen.

Subject: the role of users and providers: WG de Voogt, MD PhD, Department of Cardiology, Sint Lucas Andreas Hospital, Amsterdam, chairman; P Houdijk, Department of Cardiology, St Antonius Hospital,
Nieuwegein; HPG van Dorst, Biotronic Nederland BV, Nijmegen; Ch Kirchhof, MD PhD, Department of Cardiology, Rijnland Hospital, Leiderdorp; S Klaphake, Boston Scientific, Nieuwegein; P Stolwijk, MD, Department of Cardiology, Rijnstate Hospital, Arnhem.

Subject: costs and payment: Ms G van Oort, MSc, St Jude Medical, Veenendaal, chairman; M Siebert, St Jude Medical Bruxelles; MLL van Genugten, Medtronic Trading NL, Heerlen; VPJM Seignette, Senior Staff Officer, Financial and Management Department, Division IV, VUmc, Amsterdam.

Subject: legal aspects and responsibilities: Prof B. de Mol, MD, LLM, PhD, AMC Amsterdam, chairman; L. Bouwels, MD, Department of Cardiology, Canisius Wilhelmina Hospital, Nijmegen; PMJ Verhoeven, Department of Cardiology, St Antonius Hospital, Nieuwegein; O. Swens, LLM, Vondst Advocaten, Amsterdam; W. van den Broek, PhD, F. Böhmer, MBA; P. van Zeijst, Dutch Health Authority, The Hague, G. van Laer, Medtronic, Belgium

Subject: the role of the patient: $\mathrm{K}$ van den Broek, $\mathrm{PhD}$, Department of Psychology, University of Tilburg, chairman, Rev. PH Zaadstra, representing Dutch ICD Patient Organisation (STIN); R Derksen, MD, Department of Cardiology, Rijnstate Hospital, Arnhem; AH Maass, MD $\mathrm{PhD}$, Department of Cardiology, UMC Groningen; C Hooijschuur, Department of Cardiology, UMC Groningen; Mrs E Postelmans, RN, Amphia Hospital, Breda; H Weetink, RN, Amphia Hospital, Breda; H Versteeg, MSc, Department of Psychology, University of Tilburg; PMJ Verhoeven, Department of Cardiology, St Antonius Hospital, Nieuwegein; E. Prijs, Boston Scientific, Nieuwegein. 